\title{
BMJ Open Treatment of sarcopenia in nursing home residents: a scoping review protocol
}

\author{
Petra Benzinger (D) , ${ }^{1,2}$ Jürgen Martin Bauer, ${ }^{1,3}$ Michael Schwenk, ${ }^{3}$ Stefan Grund, ${ }^{1}$ \\ Sabine Goisser ${ }^{1}$
}

To cite: Benzinger P, Bauer JM, Schwenk M, et al. Treatment of sarcopenia in nursing home residents: a scoping review protocol. BMJ Open 2020;10:e037531. doi:10.1136/ bmjopen-2020-037531

\section{- Prepublication history and} additional material for this paper are available online. To view these files, please visit the journal online (http://dx.doi. org/10.1136/bmjopen-2020037531).

Received 06 February 2020 Revised 06 July 2020 Accepted 17 July 2020

Check for updates

(C) Author(s) (or their employer(s)) 2020. Re-use permitted under CC BY-NC. No commercial re-use. See rights and permissions. Published by BMJ.

${ }^{1}$ Center for Geriatric Medicine, AGAPLESION Bethanien

Hospital Heidelberg, Heidelberg University Hospital, Heidelberg, Germany

${ }^{2}$ Faculty of Health and Social Sciences, University of Applied Sciences Kempten, Kempten, Germany

${ }^{3}$ Network Aging Research (NAR), Heidelberg University, Heidelberg, Germany

Correspondence to Dr Petra Benzinger; petra.benzinger@bethanienheidelberg.de

\section{ABSTRACT}

Introduction Sarcopenia has been recognised as a disease that is consistently associated with a range of geriatric syndromes and negative health consequences. The prevalence of sarcopenia is high among nursing home residents. Several systematic reviews have assessed the efficacy of a range of treatment strategies against sarcopenia. However, no systematic review discussing specifically the treatment options for sarcopenic nursing home residents has been conducted so far. The objective of this scoping review, therefore, is to identify and map existing studies that assessed the feasibility and effectiveness of interventions that were conducted with the aim to treat sarcopenic nursing home residents.

Methods and analysis The protocol was developed using an established scoping review methodological framework. A systematic search of relevant literature databases will be conducted. We will also conduct a search of ClinicalTrials. gov and the WHO International Clinical Trials Registry Platform Search Portal for ongoing and recently completed trials, and will search for grey literature. Two reviewers will independently screen titles and abstracts for inclusion, followed by screening of the full text of potentially relevant articles to determine final inclusion. A data extraction sheet will be developed including key study characteristics that will be relevant for collating, summarising and reporting the results of the scoping review.

Ethics and dissemination The proposed scoping review will undertake a secondary analysis of publicly available data, and therefore does not require ethical approval. The results will be disseminated to researchers in the field by submitting the review to a peer-reviewed international journal and by presenting our findings at relevant conferences. We expect that the results of the final review will help to guide future research in the field of sarcopenia treatment for nursing home residents.

\section{BACKGROUND}

Sarcopenia refers to a condition of muscle mass loss concomitant with a loss of muscle function that exceeds the average decline as commonly seen after the age of $30-40$ years. ${ }^{1}$ Sarcopenia is consistently associated with a range of geriatric syndromes such as mobility disorders, increased risk of falls and impaired ability to perform activities of daily living. ${ }^{2-4}$ It is increasingly recognised as a disease requiring treatment in order to offset its deleterious health consequences. ${ }^{5}$
Strengths and limitations of this study

This will be the first scoping review to identify and map studies that assess the feasibility and effectiveness of interventions that have been conducted to treat sarcopenic nursing home residents.

- The review will have no time and no language restrictions; studies published in languages other than English, German, and French will be translated using an online translation tool.

- The broad scope of the review will ensure a complete overview of options to treat sarcopenia in nursing home residents that have been tested.

- The evidence obtained from the included studies, when summarised, will help to guide future research in an evolving research area with high clinical relevance.

- There will be no quality assessment of the included studies.

There is mounting evidence on the beneficial effects of exercise as the cornerstone for the treatment of sarcopenic older people. ${ }^{6}$ At the same time, evidence on the beneficial effect of nutritional interventions is less clear. ${ }^{6}$ While several pharmacological treatment options have been studied, trial results were inconsistent and, therefore, none of these is currently recommended for the treatment of sarcopenia. ${ }^{7}$

Following its first description in the 1990s, there has been an evolution of the proposed diagnostic criteria for sarcopenia. While some argue that low muscle mass alone is sufficient to diagnose it, ${ }^{8}$ in the field of geriatric research and practice today there is a consensus that for the diagnosis of sarcopenia impaired muscle function (low muscle strength and/or low physical performance) must be present together with low muscle mass. ${ }^{19-14}$ However, this consensus has only evolved in recent years and there is still considerable heterogeneity in the operationalisation of these diagnostic criteria, which in part explains the great variation in prevalence data seen. ${ }^{15}$ Such differences in the exact operationalisation aside, the prevalence of 
sarcopenia varies between different populations of older people and between different settings, with the highest prevalence of sarcopenia being reported for institutionalised older adults. ${ }^{15}$

Nursing home residents differ considerably from community-dwelling older people in their cognitive and physical capabilities as well as their medical comorbidities. ${ }^{16}$ Hence, recommendations on treatment of sarcopenia in nursing home residents should not be drawn only from studies involving community-dwelling older people. However, by fall 2019, no systematic review and no meta-analysis had been conducted to specifically establish the efficacy of interventions to treat sarcopenia in nursing home residents. Among the 27 studies included in 7 recent systematic reviews on the treatment of sarcopenic adults, ${ }^{17-23}$ only 1 study was conducted in nursing homes. ${ }^{24}$ This study was of quasi-experimental design and involved 66 participants. ${ }^{24}$ Reasons for the apparent lack of randomised-controlled intervention trials (RCTs) and even of trials with a less demanding study design concerning the treatment of sarcopenia and conducted in nursing homes might be manifold. For example, researchers might fear dilution of expected treatment effects in a nursing home population due to the fragile health and functional status of participants. ${ }^{25}$ There might also be issues related to the feasibility of interventions in this setting and challenges to attain sufficient adherence to the intervention. ${ }^{24} 26$

Notwithstanding the lack of RCTs, we are aware of trials exploring the efficacy of interventions to treat sarcopenia in nursing home residents that used designs other than randomised-controlled designs. ${ }^{24} 2728$ The need for a structured overview of all available evidence in this area is thus evident. Even if unsuitable for inclusion in systematic reviews and meta-analyses, trials using other designs than RCTs, such as uncontrolled, observational and qualitative studies, might hold information of high relevance for future research in the field. Scoping reviews are especially useful to identify and map all available types of evidence in order to facilitate future studies.

While planning this scoping review, a preliminary search for existing reviews on interventions to treat sarcopenia in nursing home residents was conducted, including the Cochrane Database, the Joanna Briggs Institute Database of Systematic Reviews and Implementation Reports, PROSPERO, Medical Literature Databases Analysis and Retrieval System Online (MEDLINE) via PubMed and the Cumulative Index to Nursing and Allied Health Literature (CINAHL). As of fall 2019, we found no review similar to the proposed scoping review.

\section{Scoping review objectives}

The objective of this scoping review is to identify and map studies that assess the feasibility and effectiveness of interventions to treat sarcopenia in nursing home residents.

\section{METHODS}

To conduct this scoping review, we will use the framework first proposed by Arksey and O'Malley, ${ }^{29}$ which has been further modified. ${ }^{30-32}$ Accordingly, our scoping review will include five stages: (1) identifying the research questions; (2) identifying potentially relevant studies; (3) selecting relevant studies; (4) charting the data and (5) collating, summarising and reporting the results. Each aspect is discussed in detail below. Preparation of this protocol was facilitated by the Preferred Reporting Items for Systematic Reviews and MetaAnalyses for scoping reviews (PRISMA-ScR) checklist. ${ }^{33}$ The initial search for relevant studies will be conducted in October 2020 and submission of results is scheduled for March 2021.

\section{Stage 1: identifying the research questions}

The objective of this scoping review is to identify studies that assess the feasibility and effectiveness of interventions for treating sarcopenia in older people residing in any kind of nursing home setting, and to map their content in order to guide future research activities. To this end, a preliminary search of the existing literature on the treatment of sarcopenia in nursing home residents was undertaken (see online supplementary appendix for preliminary search strategy used) and several research questions were developed. ${ }^{34}$ In order to define inclusion criteria, and given the heterogeneity of the studies identified, the following research questions were formulated. ${ }^{30}$ 1. What types of studies have been conducted so far in this research area or are still going on?

2. How were diagnostic criteria for sarcopenia operationalised in the conducted trials?

3. What types of interventions have been tested?

4. What outcome parameters have been used?

5. Do the tested interventions seem to be effective?

6 . What (potential) barriers to conduct such studies are reported?

7. What (potential) barriers for study participation are reported in the target population?

\section{Stage 2: identifying potentially relevant studies}

We will include studies investigating adults aged 60 years and older of either sex living in a nursing home and with a diagnosis of sarcopenia (see Stage 3 for detailed inclusion criteria). The final search strategy will be developed with the goal to maximise sensitivity. It will include terms related to the inclusion criteria (see table 1). We will not apply any age-specific search terms. We will perform an initial search of MEDLINE via PubMed and screen a limited number of retrieved articles for further relevant text words contained in the title and abstract as well as the index terms used to describe the articles. These terms will be introduced in the final search strategy. The final search strategy will then be adapted for all other databases.

We will search the following electronic bibliographic databases: 
Table 1 Population, concept and context grid showing search terms for a preliminary search of MEDLINE via PubMed

\begin{tabular}{|c|c|c|c|}
\hline & Population & Concept & Context \\
\hline Text words & $\begin{array}{l}- \\
\text { (Human adults } \\
\text { defined by } \\
\text { context/setting, } \\
\text { no age specific } \\
\text { or other search } \\
\text { terms) }\end{array}$ & $\begin{array}{l}\text { Sarcopen* } \\
\text { Dynapen* } \\
\text { Myopen* }^{*}\end{array}$ &  \\
\hline $\begin{array}{l}\text { Medical } \\
\text { Subject } \\
\text { Headings } \\
\text { terms }\end{array}$ & - & "Sarcopenia" & $\begin{array}{l}\text { "Nursing Homes" } \\
\text { "Long-Term Care" } \\
\text { "Homes for the Aged" } \\
\text { "Nursing care" } \\
\text { "Institutionalization" } \\
\text { "Residential Facilities" } \\
\text { "Housing for the } \\
\text { Elderly" }\end{array}$ \\
\hline
\end{tabular}

*Search term with truncated keywords.

- MEDLine via PubMed, including PubMed Central, in-process and other non-indexed citations, Epub ahead of print articles, author manuscripts.

- Excerpta Medica dataBase (Embase) via Ovid.

- CINAHL via EBSCOhost.

- Physiotherapy Evidence Database (PEDro).

- Web of Science, including Science Citation Index Expanded.

We will also conduct a search of ClinicalTrials.gov and the WHO International Clinical Trials Registry Platform Search Portal (ICTRP) for ongoing and recently completed trials, and will search for grey literature (ProQuest Dissertations \& Theses Full Texts, OpenGrey).

We will search all databases from inception to the present, and we will impose no restriction on language of publication or publication date. Studies published in languages other than English, German and French will be translated using an online translation tool. We will check reference lists of all primary studies and reviews identified for additional potentially eligible trials or ancillary publications. We will contact the authors of included studies and other relevant researchers in this field to seek information about further relevant published and unpublished studies. We will specifically search four key journals: Journal of Cachexia, Sarcopenia and Muscle (https:/ / onlinelibrary.wiley.com/journal/1353921906009), Journal of the American Medical Directors Association (https:/ / www.jamda. com), Journal of the American Geriatric Society (https:// onlinelibrary.wiley.com/journal/15325415) and Osteoporosis International (https://www.springer.com/journal/ 198)..$^{35}$

This scoping review will consider all randomised and quasi-randomised controlled trials (including cluster randomisation and crossover studies) as well as nonrandomised and quasi-experimental or uncontrolled trials. We will also consider retrospective and prospective observational studies, such as before-and-after and interrupted time series studies, case-control studies, case series, case reports, cohort analyses and cross-sectional studies as well as study protocols and entries in trial registries.

Search results will be imported into the Covidence software (Veritas Health Innovation, Melbourne, Australia) for removal of duplicates, screening and further reviewing.

\section{Stage 3: study selection}

Two reviewers (PB and SG) will independently conduct the screening and further reviewing using Covidence. First, they will screen title and abstract against the inclusion criteria. Articles that appear to meet the criteria or where there is any uncertainty will be obtained as full text, and both reviewers will screen the full text for inclusion. Any disagreement will be resolved through discussion or, if required, by a third person.

\section{Inclusion criteria}

For studies to be included, they should meet the following inclusion criteria:

\section{Participants}

The geriatric definition of sarcopenia is the core concept of this scoping review. In geriatric medicine, sarcopenia is defined as the age-related loss of both muscle mass and muscle function. ${ }^{19-14}$ As a consequence, for inclusion in this scoping review, studies must include participants with a diagnosis of sarcopenia based on both these aspects at baseline.

While there is no clear age limit defined for the onset of sarcopenia, loss of muscle mass and function in middle aged and younger people is very likely due to other causes than age-related sarcopenia. Within our research team, we therefore agreed to consider eligible all studies that fulfil one of the following criteria: (1) they include only participants over the age of $60,(2)$ the mean age of the sample $-1 \mathrm{SD}$ is more than 60 years or (3) results of participants aged 60 years and older are displayed separately. We will contact the study authors if in a publication there is no clear information about participant age, and ask them to provide us with separate analyses for those participants of the respective trial that are 60 years and older. In case we have been unable to obtain such information, we will present the study results indicating the uncertainty about participants' age.

\section{Context}

As outlined in the Background section, this scoping review restricts the context to the setting of nursing home 
care facilities. There are international differences in the terminology used to describe 'nursing homes'. The type and extent of care provided and the terms used to describe this care setting are heterogeneous. For the definition of 'nursing home', we will apply the operationalisation proposed by a consensus process of the International Association of Gerontology and Geriatrics (IAGG) and the American Medical Directors Association (AMDA) Foundation $^{36}$ (see online supplementary appendix). We will contact the study authors if in a publication there is no clear information to decide whether the context of the study can be regarded as a nursing home setting. If uncertainty remains, members of the research team with expertise in long-term care research will be asked to decide based on available information.

\section{Concept}

Interventions to treat sarcopenia eligible for this scoping review might include pharmacological as well as nonpharmacological strategies, such as exercise and dietary interventions. Eligible types of interventions might vary from a single delivery (eg, counselling) up to daily applications over longer periods of time (eg, dietary interventions) with variable intervention periods and even with intervals between interventions. They might address nursing home residents themselves, but also their caregivers or healthcare professionals involved in the care of nursing home residents. The interventions might be delivered as a single-type intervention or by combination of different intervention modalities (eg, exercise classes combined with counselling on physical activity) or by combinations of different intervention strategies (eg, exercise combined with a nutritional intervention) or by a combination of interventions targeting distinct target groups (eg, counselling of residents as well as healthcare professionals).

\section{Stage 4: charting the data}

Two reviewers will independently extract the data from each article included in this review. Any disagreement will be resolved through discussion or, if required, by a third person. If data on participants, interventions or study characteristics are missing or not sufficiently described, we will contact the corresponding authors and try to obtain missing information. In case the authors cannot be reached, data will be presented to the extent that is available, with a comment on what information is missing. A standardised charting form will be developed by the reviewers to collect and categorise data. The draft version includes the information listed below:

- General information: publication status, title, authors, country, year the intervention was started, year of publication (if applicable).

- Methods: study design.

- Aim of study.

- Characteristics of participants.

- Definition of sarcopenia used.

- Characteristics of the intervention.
- Primary and secondary outcome measures assessed.

- Main findings (if applicable).

- Authors conclusions (if applicable).

The drafted charting form will be updated continuously as necessary during the process of data extraction. Modifications will be detailed in the final publication.

Study quality will not be evaluated, as the primary purpose of our scoping review is to map existing evidence and to identify future areas of research for a systematic review.

\section{Stage 5: collating, summarising and reporting the results}

The search process will be described in detail by presenting a PRISMA-ScR diagram ${ }^{33}$ and stating all sources identified and screened as well as the reasons for inclusion and exclusion of all articles screened in full text. The findings of this scoping review will be presented in a tabular format as well as in a narrative summary. All identified studies will be presented detailing all characteristics relevant for answering our research questions. A descriptive narrative summary will be used to organise the key issues thematically. The findings will be discussed in terms of the overall concepts of interventions identified to treat sarcopenia in nursing home residents.

\section{ETHICS AND DISSEMINATION}

Since this review will consist of publicly available materials, it will not require any ethical approval. In line with the aim of this scoping review, we will disseminate our findings to researchers in the field by submitting the review to a peer-reviewed international journal and by presenting our findings at relevant conferences. After scoping the existing literature, we will consider a subsequent systematic review to establish the effectiveness of some of the identified interventions. We expect that the results of the final review will help to guide future research in this area.

Contributors PB and SGo conceptualised the research questions for this scoping review protocol and developed the research strategy and the methodological process. PB, SGr, MS, SGo and JMB edited and revised the research questions. PB, SGr, MS, SGo and JMB edited, revised and approved the methodological process. PB, SGo and MS drafted the manuscript. PB, SGr, MS, SGo and JMB edited and contributed to the writing of this paper, read and approved the final version and guaranteed the review.

Funding This work was supported in part by the German Federal Ministry of Education and Research (Bundesministerium für Bildung und Forschung, BMBF), grant number 01GL1732.

Competing interests JMB reported personal fees from Fresenius, personal fees from Nestlé, personal fees from Novartis, personal fees from Pfizer, personal fees from Bayer, grants and personal fees from Nutricia DANONE. He has gained no personal income from these activities and all income received has been transferred to his institution.

Patient and public involvement Patients and/or the public were not involved in the design, or conduct, or reporting, or dissemination plans of this research.

Patient consent for publication Not required.

Provenance and peer review Not commissioned; externally peer reviewed.

Open access This is an open access article distributed in accordance with the Creative Commons Attribution Non Commercial (CC BY-NC 4.0) license, which permits others to distribute, remix, adapt, build upon this work non-commercially, and license their derivative works on different terms, provided the original work is 
properly cited, appropriate credit is given, any changes made indicated, and the use is non-commercial. See: http://creativecommons.org/licenses/by-nc/4.0/.

\section{ORCID iD}

Petra Benzinger http://orcid.org/0000-0003-1121-455X

\section{REFERENCES}

1 Cruz-Jentoft AJ, Baeyens JP, Bauer JM, et al. Sarcopenia: European consensus on definition and diagnosis: report of the European Working group on sarcopenia in older people. Age Ageing 2010;39:412-23.

2 Beaudart C, Zaaria M, Pasleau F, et al. Health outcomes of sarcopenia: a systematic review and meta-analysis. PLoS One 2017;12:e0169548.

3 Schaap LA, van Schoor NM, Lips P, et al. Associations of sarcopenia definitions, and their components, with the incidence of recurrent falling and fractures: the longitudinal aging study Amsterdam. $J$ Gerontol A Biol Sci Med Sci 2018;73:1199-204.

4 Mijnarends DM, Luiking YC, Halfens RJG, et al. Muscle, health and costs: a glance at their relationship. J Nutr Health Aging 2018;22:766-73.

5 Anker SD, Morley JE, von Haehling S. Welcome to the ICD-10 code for sarcopenia. J Cachexia Sarcopenia Muscle 2016;7:512-4.

6 Dent E, Morley JE, Cruz-Jentoft AJ, et al. International clinical practice guidelines for sarcopenia (ICFSR): screening, diagnosis and management. J Nutr Health Aging 2018;22:1148-61.

7 Cruz-Jentoft AJ, Sayer AA. Sarcopenia. Lancet 2019;393:2636-46.

8 Carey EJ, Lai JC, Sonnenday C, et al. A North American expert opinion statement on sarcopenia in liver transplantation. Hepatology 2019;70:1816-29.

9 Cruz-Jentoft AJ, Bahat G, Bauer J, et al. Sarcopenia: revised European consensus on definition and diagnosis. Age Ageing 2019;48:16-31.

10 Studenski SA, Peters KW, Alley DE, et al. The FNIH sarcopenia project: rationale, study description, conference recommendations, and final estimates. J Gerontol A Biol Sci Med Sci 2014;69:547-58.

11 Fielding RA, Vellas B, Evans WJ, et al. Sarcopenia: an undiagnosed condition in older adults. current consensus definition: prevalence, etiology, and consequences. International Working group on sarcopenia. J Am Med Dir Assoc 2011;12:249-56.

12 Chen L-K, Liu L-K, Woo J, et al. Sarcopenia in Asia: consensus report of the Asian Working group for sarcopenia. J Am Med Dir Assoc 2014;15:95-101.

13 Morley JE, Abbatecola AM, Argiles JM, et al. Sarcopenia with limited mobility: an international consensus. J Am Med Dir Assoc 2011;12:403-9.

14 Muscaritoli M, Anker SD, Argilés J, et al. Consensus definition of sarcopenia, cachexia and pre-cachexia: joint document elaborated by Special Interest Groups (SIG) "cachexia-anorexia in chronic wasting diseases" and "nutrition in geriatrics". Clin Nutr 2010;29:154-9.

15 Mayhew AJ, Amog K, Phillips S, et al. The prevalence of sarcopenia in community-dwelling older adults, an exploration of differences between studies and within definitions: a systematic review and meta-analyses. Age Ageing 2019;48:48-56.

16 Onder G, Carpenter I, Finne-Soveri H, et al. Assessment of nursing home residents in Europe: the services and health for elderly in long term care (shelter) study. BMC Health Serv Res 2012;12:5.

17 Yoshimura Y, Wakabayashi H, Yamada M, et al. Interventions for treating sarcopenia: a systematic review and meta-analysis of randomized controlled studies. J Am Med Dir Assoc 2017;18:553.e1-16.

18 Vlietstra L, Hendrickx W, Waters DL. Exercise interventions in healthy older adults with sarcopenia: a systematic review and meta-analysis. Australas J Ageing 2018;37:169-83.

19 Theodorakopoulos C, Jones J, Bannerman E, et al. Effectiveness of nutritional and exercise interventions to improve body composition and muscle strength or function in sarcopenic obese older adults: a systematic review. Nutr Res 2017;43:3-15.

20 Martínez-Amat A, Aibar-Almazán A, Fábrega-Cuadros R, et al. Exercise alone or combined with dietary supplements for sarcopenic obesity in community-dwelling older people: a systematic review of randomized controlled trials. Maturitas 2018;110:92-103.

21 Hita-Contreras F, Bueno-Notivol J, Martínez-Amat A, et al. Effect of exercise alone or combined with dietary supplements on anthropometric and physical performance measures in communitydwelling elderly people with sarcopenic obesity: a meta-analysis of randomized controlled trials. Maturitas 2018;116:24-35.

22 Hsu K-J, Liao C-D, Tsai M-W, et al. Effects of exercise and nutritional intervention on body composition, metabolic health, and physical performance in adults with sarcopenic obesity: a meta-analysis. Nutrients 2019;11:2163.

23 Luo D, Lin Z, Li S, et al. Effect of nutritional supplement combined with exercise intervention on sarcopenia in the elderly: a metaanalysis. Int J Nurs Sci 2017;4:389-401.

24 Chiu S-C, Yang R-S, Yang R-J, et al. Effects of resistance training on body composition and functional capacity among sarcopenic obese residents in long-term care facilities: a preliminary study. BMC Geriatr 2018;18:21.

25 Trabal J, Forga M, Leyes $\mathrm{P}$, et al. Effects of free leucine supplementation and resistance training on muscle strength and functional status in older adults: a randomized controlled trial. Clin Interv Aging 2015;10:713.

26 Henwood T, Neville C, Baguley C, et al. Aquatic exercise for residential aged care adults with dementia: benefits and barriers to participation. Int Psychogeriatr 2017;29:1439-49.

27 Martín Del Campo Cervantes J, Habacuc Macías Cervantes $\mathrm{M}$, Monroy Torres $\mathrm{R}$. Effect of a resistance training program on sarcopenia and functionality of the older adults living in a nursing home. J Nutr Health Aging 2019;23:829-36.

28 Hassan $\mathrm{BH}$, Hewitt J, Keogh JWL, et al. Impact of resistance training on sarcopenia in nursing care facilities: a pilot study. Geriatr Nurs 2016;37:116-21.

29 Arksey H, O'Malley L. Scoping studies: towards a methodological framework. Int J Soc Res Methodol 2005;8:19-32.

30 Peters MDJ, Godfrey CM, Khalil H, et al. Guidance for conducting systematic scoping reviews. Int J Evid Based Healthc 2015;13:141-6.

31 Levac D, Colquhoun H, O'Brien KK. Scoping studies: advancing the methodology. Implement Sci 2010;5:69.

32 Levett TJ, Cresswell FV, Malik MA, et al. Systematic review of prevalence and predictors of frailty in individuals with human immunodeficiency virus. J Am Geriatr Soc 2016;64:1006-14.

33 Tricco AC, Lillie E, Zarin W, et al. PRISMA extension for scoping reviews (PRISMA-ScR): checklist and explanation. Ann Intern Med 2018;169:467.

34 Peters MDJ. In no uncertain terms: the importance of a defined objective in scoping reviews. JBI Database System Rev Implement Rep 2016;14:1-4.

35 Yang M, Tan L, Li W. Landscape of sarcopenia research (1989-2018): a bibliometric analysis. J Am Med Dir Assoc 2020;21:436-7.

36 Sanford AM, Orrell M, Tolson D, et al. An international definition for "nursing home". J Am Med Dir Assoc 2015;16:181-4. 